\title{
Preliminary Study of Temporal Change in Free Flap Volume after Tongue Reconstruction
}

\author{
Taiki Suzuki $^{1,2)}$, Ichiro Tanaka ${ }^{3,4)}$, Ryuta Osaka ${ }^{5)}$, Akira Baba ${ }^{6)}$, \\ Kazumichi Sato ${ }^{2)}$, Tomohiro Yamauchi ${ }^{(7)}$, Takeshi Nomura ${ }^{2,4)}$ and \\ Nobuo Takano ${ }^{4)}$
}

${ }^{1)}$ Department of Oral and Maxillofacial Surgery, Hachioji Hospital Tokai University, 1838 Ishikawa-cho, Hachioji, Tokyo 192-0032, Japan

2) Department of Oral Medicine, Oral and Maxillofacial Surgery, Tokyo Dental College, 5-11-13 Sugano, Ichikawa, Chiba 272-8513, Japan

${ }^{3)}$ Department of Plastic Surgery, Ichikawa General Hospital Tokyo Dental College, 5-11-13 Sugano, Ichikawa, Chiba 272-8513, Japan

${ }^{4)}$ Oral Cancer Center, Tokyo Dental College, 5-11-13 Sugano, Ichikawa, Chiba 272-8513, Japan

${ }^{5)}$ Department of Oral and Maxillofacial Surgery, Tokyo Dental College, 2-9-18 Misaki-cho, Chiyoda-ku, Tokyo 101-0061, Japan

${ }^{6)}$ Department of Radiology, Ichikawa General Hospital Tokyo Dental College, 5-11-13 Sugano, Ichikawa, Chiba 272-8513, Japan

${ }^{7)}$ Department of Oral and Maxillofacial Surgery, Tokyo Metropolitan Cancer and Infectious Center Komagome Hospital, 3-18-22 Honkomagome, Bunkyo-ku, Tokyo 113-8677, Japan

Received 1 September, 2016/Accepted for publication 7 February, 2017

\begin{abstract}
The purpose of this study was to investigate three-dimensionally temporal change in flap volume after free flap reconstructive surgery in tongue cancer patients. The results revealed an average change in flap volume of $82.3 \%$ at 1 year postoperatively. Change in tongue volume at approximately 6 months postoperatively showed a correlation with triglyceride levels. A correlation was also found between albumin levels and tongue volume at 1 year onwards postoperatively. The goal of such surgery in patients with tongue cancer is to reconstruct a functional tongue. Taking postoperative change in tongue volume into consideration is therefore of importance in minimizing postoperative dysphagia.
\end{abstract}

Key words: Oral cancer — Reconstructive operation — Volumetry — Dysphagia 


\section{Introduction}

More than 7,100 patients die of oral cancer in Japan each year, and this figure is approximately 2.7-times higher than that of 20 years ago. The number of patients with oropharyngeal cancers, included oral cancer, has been predicted to increase as the elderly population continues to rise $e^{4)}$.

Surgery and concurrent chemoradiotherapy (CCRT) are recommended as radical treatments for oral cancer $^{7}$. The appropriateness of CCRT for advanced oral cancer, however, has yet to be established, and surgery remains the primary treatment modality.

In radical surgery for oral cancer, the extent of resection depends on tumor progression, and where this is considerable, eating function and articulation may be compromised due to the subsequent decrease in the range of motion of the remaining tissue. The principal causes of postoperative dysphagia include significant postoperative change in the anatomical structures of the oral cavity and pharynx, increased sensory impairment associated with CCRT, and motor impairment due to scarring $^{12,16)}$. Many types of flap have been developed and tested for use in tongue reconstruction with the aim of mitigating such dysfunction as much as possible.

The flaps used in such reconstructive surgery can be broadly classified into two types ${ }^{14)}$ : pedicle flaps and free flaps. A pedicle flap allows grafting to be performed while maintaining a continuous blood flow. There are disadvantages with this type of flap, however, such as the limited graft range from the feeder. A free flap, on the other hand, offers no limitations on graft range and the possibility of responding to a wide range of tissue defects, the downside being that there will be complications with vascular anastomosis. For these reasons, free flap reconstruction is the most common.

One problem with the use of free flaps in reconstructing tongue is that the volume of the flap decreases over time ${ }^{2,3,6,13)}$. It is believed that this decrease is due to fatty degeneration of the muscle-fat flap itself or fusion with adi- pose tissue ${ }^{9)}$. To our knowledge, however, sufficient evidence for this hypothesis has yet to be provided.

In some cases, this temporal decrease in the volume of reconstructed tissue makes it difficult to maintain the ability to perform the motions needed for eating and swallowing, even with postoperative rehabilitation. This dysphagia causes a marked deterioration in postoperative quality of life (QOL).

The purpose of this study was to determine the effect of temporal change in volume of reconstructed tongue on eating and swallowing function, together with that on articulatory function. This was achieved by using computed tomography (CT) to determine the kinetics of temporal decrease in the volume of grafted flaps during postoperative follow-up.

\section{Materials and Methods}

\section{Patients}

The study protocol conformed to the principles outlined in the Declaration of Helsinki and was approved by the Ethics Review Board of Tokyo Dental College Ichikawa General Hospital (approval number: 116-35).

A total of 50 patients undergoing reconstructive intervention for various types of defect of the oral cavity and neck at the Oral Cancer Center of Tokyo Dental College (Chiba, Japan) between July 2006 and March 2013 were included in the study. According to established NCCN guidelines ${ }^{4}$, , surgical resection is the standard therapy in patients with operable oral cancer. Among the 50 patients in the present study, various types of pedicle flap were used in 4 and various types of free flap in the remaining 46 . Free flaps were used for the tongue in 25 patients, for the lower gingiva in 10 patients, for the floor of mouth in 10 patients, and for the buccal mucosa in 1 patient. Of these, we selected 10 patients from the 25 with tongue cancer. The observation period was between 12 and 22 months postoperatively (median, 15 months). Postoperative rehabilitation for dysphagia is performed as a 

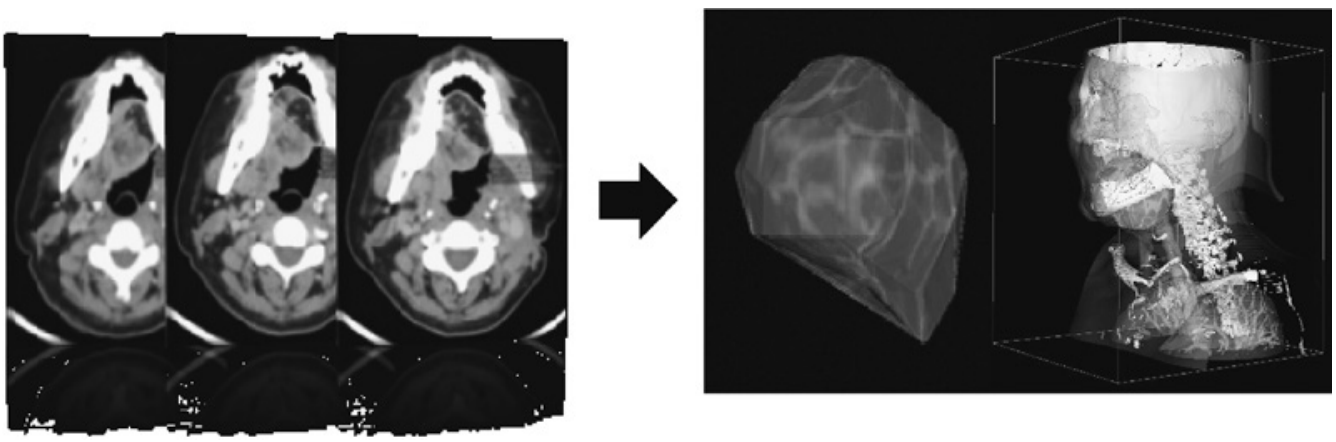

Fig. 1 Method for calculating volume of reconstructed tongue

A radiodiagnostician selected area of reconstructed tongue as region of interest (ROI); volume of ROI, which was automatically configured from there, was calculated.

matter of course at our department in such patients.

\section{Methods}

Postoperative CT was used for retrospective evaluation (slice width, $3 \mathrm{~mm}$ ). Change in flap volume was determined with DICOM image processing software $\left(\right.$ OsiriX $^{\circledast}$, OsiriX Foundation, Geneva, Switzerland). A radio diagnostician selected the area of the reconstructed tongue to be used as the region of interest (ROI) and calculated the volume of the ROI, which was automatically configured from there (Fig. 1). A uniform margin of $5 \mathrm{~mm}$ was set up on the side of the reconstructed tongue that was sutured.

1) Items surveyed

The following data were extracted from the patients' medical records: TNM classification, type of flap, age, surgical procedure, number of angiostomies, time elapsed postoperatively, body weight, body mass index (BMI), and nutritional status. Potential correlations between each of these factors and change in flap volume were then determined statistically with the Spearman's product-moment correlation coefficient test. Statistical significance was set at $\mathrm{p}<0.05$.

2) Items evaluated

The primary endpoint was time elapsed postoperatively and change in volume of reconstructed tongue. The secondary end- point was factors associated with change in volume of reconstructed tongue.

\section{Results}

\section{Patient characteristics (Table 1)}

The patients' mean age was 56.9 years (range, 30-72 years). The size of the primary tumor was cT2 in 1 case, cT3 in 2, and cT4 in 7. In terms of cervical lymph node metastasis, there were 3 cases of $\mathrm{cN} 0,3$ of $\mathrm{cN} 1,1$ of $\mathrm{cN} 2 \mathrm{a}$, and 3 of cN2b. In terms of type of surgical procedure performed for primary lesion resection, 7 patients underwent a partial glossectomy, and 3 underwent subtotal glossectomy. In terms of surgical procedure used for cervical lymphadenectomy, 8 patients underwent unilateral neck dissection and 2 underwent bilateral neck dissection. Free forearm flaps (FF) were used in 8 cases and free rectus abdominal myocutaneous flaps (RAMF) in 2 cases. Finally, in terms of angiostomies, each flap contained one artery and an average of 2 veins (range, $1-5$ veins).

\section{Change in volume of reconstructed tongue (Fig. 2, Table 1)}

The average rate of change in volume of CT2 on radiography at approximately 6 months postoperatively was $79.5 \%$ (range, $53.1-107 \%)$. The average rate of change in 
Table 1 Items surveyed

\begin{tabular}{ll}
\hline \hline Age & 30-72 (Ave. 56.9) \\
T stage & T2 (1 case), T3 (2 cases), T4 (7 cases) \\
N stage & N0 (3 cases), N1 (3 cases), N2a (1 case), N2b (3 cases) \\
Primary resection & HG (7 cases), STG (3 cases) \\
Neck dissection & UL (8 cases), BL (2 cases) \\
Angiostomy artery/vein & $1 / 1-5$ (Ave. 2) \\
Preoperative Albumin levels & $3.8-5.0 \mathrm{~g} / \mathrm{dl}$ (Ave. $4.4 \mathrm{~g} / \mathrm{dl})$ \\
Preoperative Total Protein levels & $6.2-7.5 \mathrm{~g} / \mathrm{dl}$ (Ave. $7.0 \mathrm{~g} / \mathrm{dl})$ \\
Preoperative Triglyceride levels & $41-191 \mathrm{mg} / \mathrm{dl}$ (Ave. $113 \mathrm{mg} / \mathrm{dl})$ \\
Preoperative BMI & $19.6-36.0$ (Ave. 26.1) \\
Base study Volume Ratio of CT2 & $53.1-107 \%$ (Ave. 79.5\%) \\
Base study Volume Ratio of CT3 & $51.4-132 \%$ (Ave. 82.3\%) \\
Base study Volume Ratio of CT4 & $44.1-140 \%$ (Ave. 83.4\%) \\
\hline
\end{tabular}

HG: half glossectomy, STG: subtotal glossectomy, UL: unilateral, BL: Bilateral.

Average rate of volume change of CT2 on radiography at approximately 6 months postoperatively was $79.5 \%$ (range, 53.1-107\%). Average rate of volume change of CT3 on radiography at approximately 1 year postoperatively was $82.3 \%$ (range, $51.4-132 \%$ ).

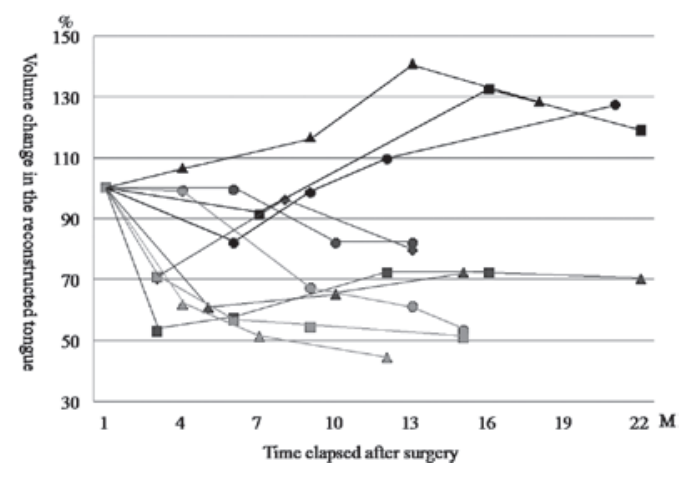

Fig. 2 Amount of variation in tongue volume over time after tongue reconstruction

Range of volume change at approximately 6 months postoperatively was $53.1-107 \%$. Range of volume change at approximately 1 year postoperatively was $51.4-132 \%$.

volume of CT3 on radiography at approximately 1 year postoperatively was $82.3 \%$ (range, 51.4-132\%).

\section{Items correlated with change in volume}

(Fig. 3)

No correlation was found between change in volume and TNM classification, surgical

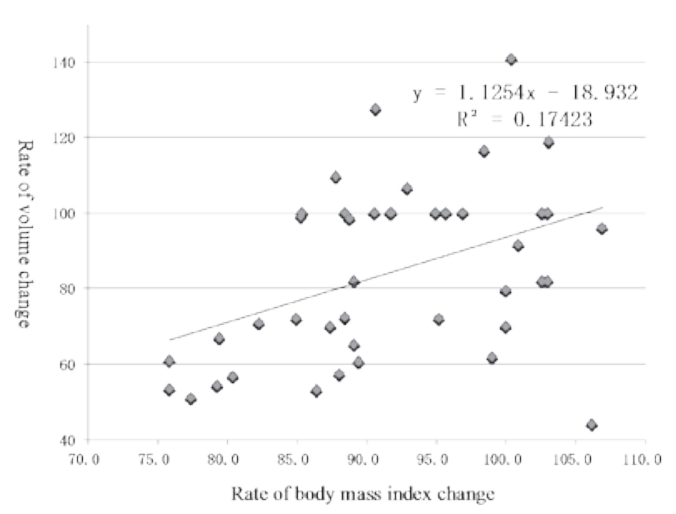

Fig. 3 Correlation between body mass index and volume of reconstructed tongue

Although body weight and body mass index (BMI) were presumed to be correlated with volume of reconstructed tongue, no such correlation was observed.

procedure, anastomosed blood vessels, body weight, or BMI, all of which are generally thought to be associated with change in volume of reconstructed tongue.

Significant differences were found in change in volume of reconstructed tongue and preoperative triglyceride levels $(\mathrm{p}<0.025)$ and albumin levels $(\mathrm{p}<0.032, \quad \mathrm{p}<0.010)$ 
Table 2 Spearman's product-moment correlation coefficients

\begin{tabular}{|c|c|c|c|c|}
\hline & & $\begin{array}{l}\text { CT2 Base study } \\
\text { Volume Ratio }\end{array}$ & $\begin{array}{l}\text { CT3 Base study } \\
\text { Volume Ratio }\end{array}$ & $\begin{array}{c}\text { CT4 Base study } \\
\text { Volume Ratio }\end{array}$ \\
\hline \multirow[t]{2}{*}{ Age } & $\mathrm{CO}$ & -0.16 & 0.13 & 0.21 \\
\hline & SP & 0.66 & 0.72 & 0.57 \\
\hline \multirow[t]{2}{*}{ Preoperative Albumin levels } & $\mathrm{CO}$ & 0.46 & 0.68 & 0.76 \\
\hline & SP & 0.19 & $* 0.03$ & $* 0.01$ \\
\hline \multirow[t]{2}{*}{ Preoperative Total Protein levels } & $\mathrm{CO}$ & 0.48 & 0.51 & 0.49 \\
\hline & $\mathrm{SP}$ & 0.16 & 0.14 & 0.15 \\
\hline \multirow[t]{2}{*}{ Preoperative Triglyceride levels } & $\mathrm{CO}$ & 0.70 & 0.61 & 0.44 \\
\hline & $\mathrm{SP}$ & $* 0.03$ & 0.06 & 0.20 \\
\hline \multirow[t]{2}{*}{ Preoperative BMI } & $\mathrm{CO}$ & 0.43 & 0.22 & 0.16 \\
\hline & $\mathrm{SP}$ & 0.21 & 0.53 & 0.65 \\
\hline \multirow[t]{2}{*}{ 1st BMI Ratio } & $\mathrm{CO}$ & 0.10 & -0.04 & -0.02 \\
\hline & $\mathrm{SP}$ & 0.80 & 0.91 & 0.96 \\
\hline \multirow[t]{2}{*}{ 2nd BMI Ratio } & $\mathrm{CO}$ & 0.05 & 0.47 & 0.48 \\
\hline & $\mathrm{SP}$ & 0.90 & 0.21 & 0.19 \\
\hline \multirow[t]{2}{*}{ 3rd BMI Ratio } & $\mathrm{CO}$ & 0.14 & 0.57 & 0.69 \\
\hline & $\mathrm{SP}$ & 0.74 & 0.14 & 0.06 \\
\hline \multirow[t]{2}{*}{ 4th BMI Ratio } & $\mathrm{CO}$ & 0.07 & 0.18 & 0.26 \\
\hline & $\mathrm{SP}$ & 0.86 & 0.63 & 0.47 \\
\hline
\end{tabular}

$* \mathrm{p}<0.05$ CO: correlation odds, SP: significance probability

Statistically significant differences were observed in preoperative triglyceride $(\mathrm{p}<0.025)$ and albumin levels $(p<0.032, p<0.010)$. When preoperative triglyceride levels were high, flap volume was less likely to decrease during early postoperative period (approximately 6 months postoperatively). When preoperative albumin levels were high, volume was less likely to decrease during late postoperative period (at 1 year onwards postoperatively).

(Table 2). When preoperative triglyceride levels were high, the flap volume was less likely to decrease during the early postoperative period (about 6 months postoperatively), and when preoperative albumin levels were high, the volume was less likely to decrease during the late postoperative period (at 1 year onwards postoperatively).

\section{Discussion}

In an earlier study on change in volume of transplanted flaps, the surface area of the reconstructed tongue was calculated by multiplying the width of one slice of imaging data by the number of slices ${ }^{9}$. Meanwhile, in another study, change in the height of the reconstructed tongue in the sagittal section was evaluated $^{12,17)}$. While the methods used in these earlier studies allowed for calculating the ratio of change in volume of reconstructed tongue, the estimated findings differed from the actual volume ${ }^{5,9)}$. In order to accurately evaluate such change, therefore, it is necessary to accurately assess tongue volume. The present study has attempted to address this issue.

Here, we selected the OsiriX ${ }^{\circledR}$. This has been shown to guarantee accuracy in calculating volumes in liver resection and precision in terms of reproducibility of tissue volume ${ }^{1)}$. However, the amount of tissue involved in reconstructed tongue is smaller than that involved in the liver. In the above-mentioned study on liver resection, 5-mm CT slices were 
used; however, in order to adapt the technique to the oral area, the CT slices were set to $3 \mathrm{~mm}$ in the present study. This enabled accurate assessment with both the thin-type free flaps and thicker RAMFs. The plasticity of methods for analyzing flap volume using OsiriX ${ }^{\circledast}$, however, remains to be established in studies of oral cancer. Furthermore, one limitation of the present study was that the fill rate of the grafted tissue was not unified, which meant that change in volume of grafted tissue could only be observed in isolation.

Temporal change in reconstructed tongue revealed that volume decreased in some cases while it increased in others. The transplanted free flaps were of the muscle-fat type, so the observed increase in some cases may have been associated with fatty degeneration of muscle. Rihani et $a l^{8)}$ and Sakamoto et $a l^{9}{ }^{9}$ also suggested that change in muscle and fat tissue in transplanted flaps was related to change in flap volume.

In a previous report, Fujioka et $a l^{2}{ }^{2}$, Siebert et $a l^{10)}$ and Siebert and Longaker ${ }^{11)}$ reported considerable change in amount of tissue with rectus RAMFs. In another report ${ }^{15)}$, however, Yamaguchi et al. reported that such change was absent in RAMFs and anterolateral thigh flaps. In the present study, only FFs and RAMFs were investigated. The patterns of change in volume of reconstructed tongue did not appear to be specific to type of flap.

In future study, this group intends to use previous reports as a reference in investigating temporal change in muscle and adipose tissue in reconstructed tongue.

The present results revealed a correlation between preoperative triglyceride levels and change in volume of reconstructed tongue during the early postoperative period (at approximately 6 months). This correlation suggests that early change in volume of reconstructed tongue is influenced by the amount of muscle and fat on the floor of the tongue.

The present findings also indicate that at 1 year postoperatively onwards, change in volume of reconstructed tongue is associated with preoperative albumin levels, suggesting that postoperative nutritional status is corre- lated with change in flap volume. No correlation was observed, however, with BMI or body weight. Further study is needed to investigate such change in relation to postoperative nutritional status.

The surgical procedures and number of angiostomies to the flap were also investigated, but the findings revealed no association with change in volume of reconstructed tongue.

A number of studies have investigated the relationships between reconstructed tongue and articulatory and swallowing functions, but there have been various issues regarding reproducibility, and the details remain unclear. A truly objective evaluation of decrease in volume of tissue would help establish functional reconstruction methods capable of providing oral cancer patients with a higher QOL; accompanying surgical treatments that could provide a radical cure; and predictors of postoperative disorders.

This group is currently studying the relationships among change in volume of reconstructed tongue, chronological variation in nutritional status, and swallowing function. A potential association between postoperative CCRT and postoperative infection is also being investigated, although this was outside the scope of the present study.

\section{Conclusion}

Three-dimensional imaging was used to investigate temporal change in flap volume after free flap transplantation in tongue cancer patients. The results revealed an average change in volume of $82.3 \%$ at approximately 1 year postoperatively. Change in volume at approximately 6 months postoperatively showed a correlation with triglyceride levels, whereas change at 1 year onwards showed an association with albumin levels. We believe that a greater understanding of change in postoperative volume would allow improved functionality in cases of tongue reconstruction. 


\section{References}

1) D’Onofrio M, De Robertis R, Demozzi E, Crosara S, Canestrini S, Pozzi Mucelli R (2014) Liver volumetry: Is imaging reliable? Personal experience and review of the literature. World J Radiol 6:62-71.

2) Fujioka M, Masuda K, Imamura Y (2011) Fatty tissue atrophy of free flap used for head and neck reconstruction. Microsurgery 31:32-35.

3) Hallock GG (2004) Long-term superiority of composite versus muscle-only free flaps for skull coverage. Ann Plast Surg 52:507-510.

4) Japan Society for Head and Neck Cancer Registry Committee (2006) Report of head and neck cancer registry of Japan. Clinical statistics of registered patients, 2002. Japanese J Head and Neck Cancer 32:15-34.

5) Joo YH, Hwang SH, Park JO, Cho KJ, Kim MS (2013) Functional outcome after partial glossectomy with reconstruction using radial forearm free flap. Auris Nasus Larynx 40: 303-307.

6) Kimata Y, Sakuraba M, Hishinuma S, Ebihara S, Hayashi R, Asakage T, Nakatsuka T, Harii K (2003) Analysis of the relations between the shape of the reconstructed tongue and postoperative functions after subtotal or total glossectomy. Laryngoscope 113:905-909.

7) National Comprehensive Cancer Network (2016) NCCN Guidelines, Version 1. Panel Members Head and Neck Cancers, https:// www.nccn.org/professionals/physician_ gls/f_guidelines.asp (5 October, 2017)

8) Rihani J, Lee MR, Lee T, Ducic Y (2013) Flap selection and functional outcomes in total glossectomy with laryngeal preservation. Otolaryngol Head Neck Surg 149:547-553.

9) Sakamoto Y, Takahara T, Ota Y, Aoki T, Yamazaki H, Otsuru M, Takahashi M, Aoyama K, Kaneko A, Kawada S, Ichikawa T, Imagawa K, Miyasaka M (2014) MRI analysis of chronological changes in free-flap volume in head and neck reconstruction by volumetry. Tokai J Exp Clin Med 39:44-50.
10) Siebert JW, Anson G, Longaker MT (1996) Microsurgical correction of facial asymmetry in 60 consecutive cases. Plast Reconstr Surg 97:354-363.

11) Siebert JW, Longaker MT (2001) Aesthetic facial contour reconstruction with microvascular free flaps. Clin Plast Surg 28:361-366.

12) Tei K (2005) Rehabilitation program for postoperative swallowing dysfunction in patients with oral squamous cell carcinoma. Toukeibu Gan 31:308-312. (in Japanese)

13) Wolff KD, Stiller D (1992) Functional aspects of free muscle transplantation: atrophy, reinnervation, and metabolism. J Reconstr Microsurg 8:137-142.

14) Wong CH, Wei FC (2010) Microsurgical free flap in head and neck reconstruction. Head Neck 32:1236-1245.

15) Yamaguchi K, Kimata Y, Onoda S, Mizukawa N, Onoda T (2012) Quantitative analysis of free flap volume changes in head and neck reconstruction. Head Neck 34:1403-1407.

16) Yamamoto N, Mitsudo K, Hayashi Y, Tohnai I, Ueda M (2000) Postoperative oral functional disturbance and socioeconomic changes in the life of the oral cancer patient. Toukeibu Shuyou 26:57-62. (in Japanese)

17) Yamazaki Y, Tei K, Makino S, Kitada H, Ueno T, Moriyama S, Notani K (2003) Decrease in the free flap volume caused deterioration of swallowing function after subtotal glossectomy in a patient with tongue cancer: a case report. Nihon Sesshoku Enge Rihabiritēshon Gakkai Zasshi 7:159-165. (in Japanese)

\section{Correspondence:}

Dr. Taiki Suzuki

Department of Oral and

Maxillofacial Surgery,

Hachioji Hospital Tokai University, 1838 Ishikawa-cho, Hachioji, Tokyo 192-0032, Japan E-mail: suzukitaiki@tdc.ac.jp 